\title{
Construction of Urban Commercial Fitness and Leisure Space from the Perspective of Emotional Geography
}

\author{
Baorong $\mathrm{Li}^{1 *}$, Huimin $\mathrm{Bao}^{1}$ \\ ${ }^{1}$ Tourism\&Institute of Human Geography, Xi'An International Studies University, Xian, Shaanxi, 710128, China \\ *Corresponding author's e-mail:107242019200236@stu.xisu.edu.cn
}

\begin{abstract}
As people pay more and more attention to the shaping of healthy body, the current gym, a commercial sports place, has developed rapidly and become a new leisure space in modern society. The themes of geographical imagination, local identity and spatial production in fitness and leisure space began to attract scholars' attention. By reviewing and analyzing the relevant literature, it is found that deconstructing the spatial emotional dimension is a geographical research method that can naturalize the gender differences in fitness and leisure activities. The gym is used as an indoor environment to understand and intervene the broader gender differences in fitness and leisure activities, and analyze and explore how emotions affect individuals' interaction in their environment, this will help us find and narrow the gender differences in space. Based on the theory of emotional geography and feminist geography, this paper analyzes the participants' emotional experience of fitness and leisure activities, and points out that the relationship between participants' fitness and leisure behavior socially constructs space. The emotional experience of fitness and leisure behavior is highly related to the condensation and influence of discourse power.
\end{abstract}

Keywords : Emotional geography, Urban commercial fitness and leisure space, Construction

\section{INTRODUCTION}

Under the influence of "emotional turn", geographers believe that emotion is an important dimension of building space in the process of society, economy, politics and even power ${ }^{[1]}$. In view of the high gender nature of fitness and leisure activities and the inequality of gender and other social factors in participation for a long time, it is necessary to adopt the method of feminist geography to study in the fitness and leisure activity space centered on the "society space power" structure, so as to bring emotion into the space of sports and fitness activities, It helps to reveal the role of gender inequality in spatial reproduction and provide more safe and barrier free opportunities for women and other vulnerable groups to participate in fitness and leisure.

The gender research of emotional geography involves the discussion of the relationship between male power and female emotion, and the emotional characteristics and changes of women in specific time and daily life space. In the study of women's fear emotion, the relationship between women's fear emotion and patriarchal space ${ }^{[2]}$ and the environment that causes women's fear emotion [3]; Some scholars focus on women's emotions from their daily space such as eating habits, shopping consumption and weekend life ${ }^{[1]}$; Nina explores the relationship between emotion and gender, sex and "race" in night leisure space, and makes contributions to the field of emotional geography and female geography ${ }^{[4]}$.

Fitness space is a space that is easy to cause tension. Some scholars even describe the gym as a "fear space" [5], especially women experience psychological problems related to worry and anxiety caused by physical comparison and evaluation in the gym [6] Johansson's research on female bodybuilders has aroused geographers' attention to the dual space of body gender and gym environment gender ${ }^{[7]}$. In the existing gender research, the focus has been on professional bodybuilding gyms and gyms for prenatal exercise for pregnant women, and less attention has been paid to the gender differences of non-competitive fitness activities and geographical emotional experience. Although critical views on gender have been put forward in 
leisure sports activities such as surfing, walking and roller skating ${ }^{[8]}$, emotional geographers have not carried out gender research in fitness leisure space. Emotional geography mainly focuses on how time and space relate to emotion and act on people's emotional changes, trying to enrich the theoretical exploration of human behaviour and activity space through emotional intermediary. At present, foreign research has become mature and has initially formed a system. However, there is little research on emotional geography in China, and the existing ones focus on the introduction of basic theories; in practice, there are many studies on the positive emotional relationship between local attachment and local sense, but there is still a lack of attention in emotional space and sociality.

With the increasing leisure time of urban residents, participating in fitness and leisure activities has become one of the main leisure activities in urban residents' daily life. The public space specially used for fitness in urban secondary schools has gradually sprung up. Urban fitness and leisure space is an important part of urban leisure space, a place for urban residents to carry out fitness activities and a sign of urban humanization. Geographers should study the flow of elements of indoor space and the way of mutual connection, because indoor space occupies a central position in people's daily life, and indoor intervention behaviour can have repercussions on different spatial scales and change the relationship between man and environment, which is helpful to explain the relationship between man and environment, Instead of simply describing the interaction between human and environment in indoor space ${ }^{[9]}$. The study of how indoor space is connected with social ecosystem can have a deeper understanding of previous studies and produce new geographical problems. From this point of view, the gym can be positioned as a fitness leisure space for daily sports fitness activities. It is a space directly affected by the gender of sports activities and may have a more general gender impact.

Fitness is a kind of non-competitive social sports for the public with health as the goal. Yoga, aerobics, iron lifting and other fitness methods have long become an important part of contemporary people's daily life. Gym is a common place for daily fitness and leisure activities. It is considered to be a highly masculine space. The gender inequality in the space has a potential role in strengthening gender differences ${ }^{[10]}$. Therefore, based on the perspective of emotional geography, this paper studies the gender differences in the gym space, tries to deconstruct its emotional space, and puts forward suggestions for the gym to build a good emotional space, so as to improve the embarrassing situation of gender inequality.
This study is based on the intersection of fitness and leisure activities, emotional geography and feminist geography. Through reviewing and sorting out the relevant studies, it is found that the research based on emotional geography and combined with the relevant theories of feminist geography is a geographical research method that can naturalize the gender differences in fitness and leisure activities. In the study of feminist geography, bringing emotion into the space of sports fitness activities is complementary to the cross empirical study of emotional geography and female geography. The study of emotional geography is helpful to the construction of liveable, happy and healthy cities in China, and is of great significance to social harmony and sustainable development. Invisible affective factors allow and restrict the participation of physical activities, and play a role in revealing the emotion in the gender unequal relationship of spatial reproduction. Therefore, it is necessary to adopt a more critical geographical method for research in the fitness and leisure activity space centered on the "society space power" structure, so as to provide more safe and barrier free fitness and leisure space and participation opportunities for vulnerable groups such as women, so as to improve their overall participation level in sports activities, so as to help narrow the gender and regional gap, It is of great significance to provide fair fitness and leisure space and health opportunities for cities.

\section{MATERIALS AND METHODS}

This research was conducted in the gym in South $\mathrm{Xi}$ 'an. This article mainly uses research methods such as time geography, questionnaire surveys, participatory observations, semi-structured interviews and activity diaries to collect and statistically process data. Respondents' criteria included: members of the gym and regular visitors, participating in daily exercises in strength and/or "aerobic" areas, and ages between 25 and 64. By establishing an emotional indicator system, interviewing respondents and filling in activity diaries, focusing on the respondents' relationship with the gym, their gym environment, negative and positive gym experiences, and their perceptions of gym gender, it analyzed the impact of gender factors on different feelings. The degree of influence of visitors' emotional experience, summarizing the influence of gender on emotional experience in the gym space.

\section{RESULTS \& DISCUSSION}

Gym is a place where the idealization and stereotype of gender body can be embodied. These gender characteristics are reflected in the material structure of gym and in personal practice. 
Table 1. Gender performance in the gym.

\begin{tabular}{ccc}
\hline & Woman & Man \\
\hline Space & Tend to choose gyms around residential areas & Tend to choose a gym outside the residential area \\
requirement & & \\
Time and space Family responsibilities and social role positioning areHigh frequency of using fitness and leisure space \\
constraints & unique restrictive factors for female groups & "Strength" anaerobic zone and weightlifting zone \\
Gender space & "Slimming" aerobic zone and aerobics room &
\end{tabular}

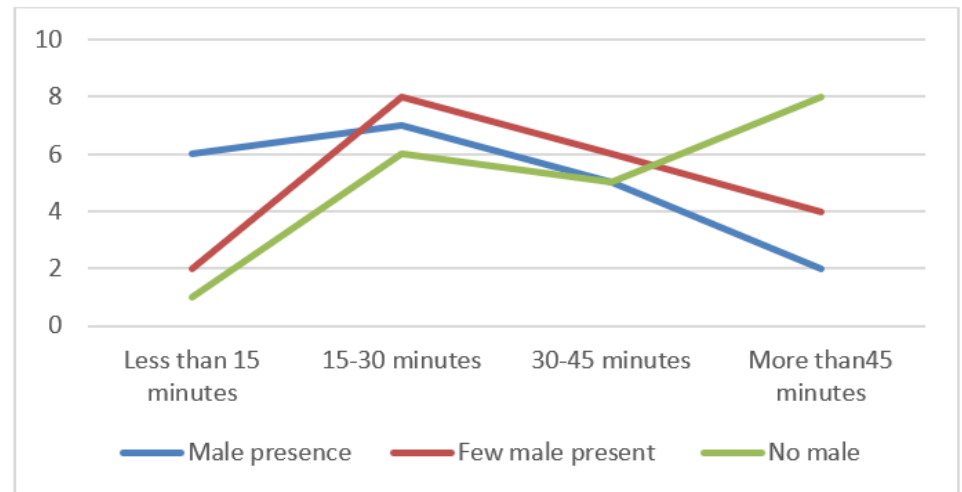

Figure 1. The influence of gender factors on women's exercise time

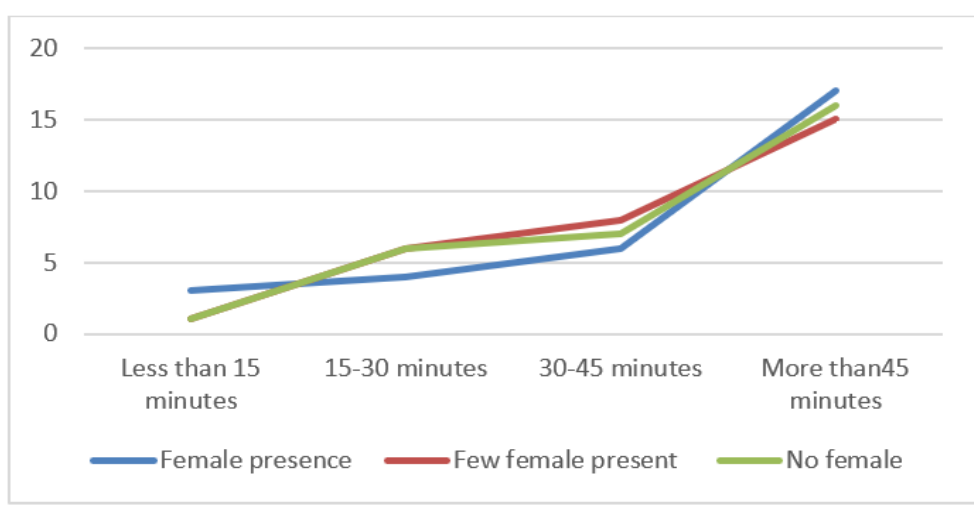

Figure 2. The influence of gender factors on men's exercise time

Gym can be described as a typical space where male and female body cultures often coexist, that is, the intersection of fitness culture and aerobics culture. The gender relationship in the gym will be reflected in clothing, behaviour, facilities and posters. The concept of geographical imagination of the gym is rooted in the level of material experience (i.e. body and dress). In this way, material can actively occupy the psychological social space. Studying these spaces can understand women's experience in the gym and how the gender performance of the gym affects fitness leisure behaviour.
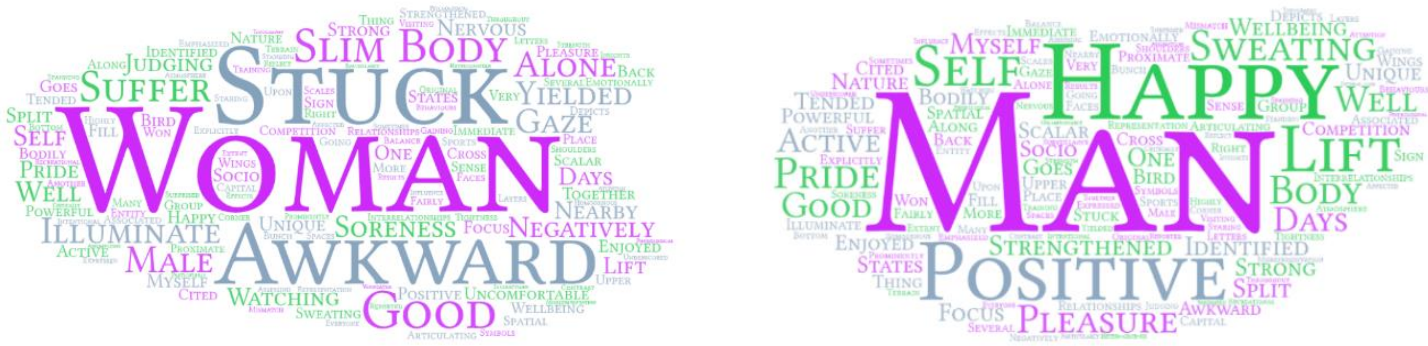

Figure 3. Word frequency diagram created from male and female respondents' descriptions of gender influence 
The fitness environment produces three emotional experiences about gender relations, namely dislocation tension, anxiety and gender gaze caused by self and others' evaluation. They jointly construct the Unbalanced Gender emotional structure of the place, so that the boundary of the local hierarchical structure of masculinity and feminization is felt in the way of generating negative emotions (such as tension and anxiety), This in turn strengthens the gender boundaries for participation in sports fitness and leisure activities [10].

\subsection{Spatial dislocation}

People will default that some spaces and experiences in the gym are men's spaces. The masculinity of these spaces is so deep-rooted that some women call the weight lifting area of the gym "boys' Club" or "men's area", and regard the women entering the area as strange. Many studies on gender identity in gyms show that the increased polarization between women and men is related to the tension embedded in a series of behaviors based on the placement of gender relations ${ }^{[11]}$. Both women and men experience an anxious sense of gender dislocation, because they realize that their masculinity or femininity is inconsistent with or even subordinate to the dominant men or women in the gym environment, which leads to the gap in sense of belonging. Many women attribute their discomfort to the collision they feel when their femininity conflicts with masculine space. The gender hierarchy of the gym highlights the male hegemony, which is the default gender in the gym space, making the femininity incompatible with other masculinity.

\subsection{Supervision and evaluation}

Gym space can lead to negative self-evaluation and anxiety about being negatively evaluated by others. In the gym, men and women don't have any inherent clothes, but reflect the masculinity and femininity in a specific context and the relationship between them. The gym stipulates a specific standard femininity, which is reflected in the slim physique wearing suitable clothes, by indicating which women's bodies are accepted by the society in the gym. The unwritten regulations on what women should wear in the gym are so strong that departing from this standard will damage women's comfort and self-esteem. Although they are proud to improve their health level, the clothes in the gym bring dissatisfaction with their body.

Compared with other spaces, women in the gym are more vulnerable to male gaze, especially considering that men monitor themselves and each other in the gym, women often look at each other in the space marked as women (i.e. gym) ${ }^{[12]}$. For many women, the physical appearance compared with other gyms is the source of negative emotions. They think they don't reflect the typical gyms' femininity. Studies have shown that the existence of mirrors "supports a continuous monitoring state, including the monitoring of themselves and others", which means that everyone has the opportunity to see others without the other party knowing that they are being monitored [13]. Mirrors produce self-monitoring, both obvious and hidden. Therefore, self-monitoring is not only a gender relationship, but also a function of a specific gym space.

\subsection{Gender gaze}

Some theories believe that women will look at themselves from the perspective of men in the gym, especially in the male dominated space of the gym, but men's gaze has different meanings ${ }^{[14]}$. Some women said that male gaze and interaction would interfere with their exercise and activities in the gym. This model is consistent with Waitt's research results, that is, women in surfing space will be affected by male gaze ${ }^{[15]}$. Women are self-conscious and will avoid being stared according to the ongoing exercise and the position of their body or clothing. This special situation can be understood from the more general gender order: Men's eyes express the desire to dominate, while the different strategies formulated by young women are interpreted as resistance to trying to define and subordinate. To some extent, the body of young female bodybuilders is affected by the current male aesthetic orientation, which can be regarded as an external discipline. However, when this power is applied to women's body, we should also pay attention to the individual initiative. Women's fitness practice and its physical "counter attack" are realized in the interweaving process of a variety of emotions. In short, women's bodies are not only disciplined by external forces, but also the space for the expression of their own emotions and temperament, but also the embodiment of their self-worth and vitality.

\section{CONCLUSIONS}

Driven by commercialization, the gym space that used to prefer masculinity is changing. Nowadays, women are increasingly interested in joining professional women's gyms and leisure clubs. These facilities have become another "battlefield" for women to pursue fashion, attitude, health, beauty and self-improvement. The expansion of women's commercial fitness and leisure space has provided some women with more colorful life. These women have changed their appearance and social status and built their own development space by consuming fitness and leisure products. On the other hand, this type of commercial space also plays a role of differentiation and exclusion, because women who can't afford this kind of consumption are excluded from this space. 
Commercial fitness and leisure space meets the needs of modern women in the pursuit of material, spiritual and social status. Through consumption, women have become the main target group of the fitness industry under neoliberalism, thus reshaping the boundary of gender space. Women are not only regarded as the subject of consumption, but also as the symbol of consumption related to materialism. Commercial space is full of female culture. The expansion of fitness and leisure space in commercial area is related to the trend of "feminization". Women are potentially important customers because they care more about their appearance than men. As female members prefer to participate in collective aerobics courses rather than use fitness equipment, the gym began to change its business strategy and launched more projects suitable for women, such as reducing the equipment area, increasing the aerobic area and yoga room, and decorating the gym with feminine colors to make the exercise environment comfortable and pleasant, so the number of female members increased sharply.

Fitness and leisure space may be a place for women's health, well-being, social network and collective empowerment. Deconstructing its emotional dimension is a geographical research method that can naturalize gender differences in fitness and leisure activities. Women's fitness and leisure behavior relationship socially constructs gender relations, the emotional experience of fitness leisure behavior is highly related to the gaze and influence of discourse power. Women's fitness and leisure space reflects the diversity and dynamics of women's space, which also means that women have sustainable social power in fitness and leisure places, but there may be gender differences and class stratification. In the future feminist geography research, bringing emotion into the spatial area of fitness and leisure activities will help to reveal a more profound problem that is, the role of place in (RE) generating gender health inequality, and have an impact on the geographical research of health and social justice.

\section{ACKNOWLEDGMENTS}

This paper is one of the phased achievements of the graduate fund key project of Xi'an Foreign Studies University, "gender relationship construction of urban fitness and leisure space from the perspective of emotional geography -- Taking Xi'an operational gym as an example" (SSZD2020028).

\section{REFERENCES}

[1] Jian J, Zhen F, Xi GL, Zhai Q. (2016) Research progress and Enlightenment of Western emotional geography. Research on World Geography, (02):123-136

[2] Gill V. (1989) The Geography of Women's Fear.
Area, (4):385-390

[3] Pain R. (1991) Space, sexual violence and social control: integrating geographical and feminist analyses of women's fear of crime. Progress in Human Geography, 15(4): 415-431.

[4] Held N. (2015) Comfortable and safe spaces? Gender, sexuality and 'race' in night-time leisure spaces. Emotion, Space and Society, 33-42.

[5] Frew, M., McGillivray, D. (2005) Health clubs and body politics: Aesthetics and the quest for physical capital. Leisure Studies, 24, 161-175.

[6] Pridgeon, L., Grogan, S. (2012) Understanding exercise adherence and dropout: An interpretative phenomenological analysis of men and women's accounts of gym attendance and non-attendance. Qualitative Research in Sport Exercise and Health, $4,1-18$.

[7] Johansson T. (1996) Gendered spaces: The gym culture and the construction of gender. Young, Vol.4 (3):32-47.

[8] Gavin J. A, Mark I. Sudwell S, Andrew C. (2005) Towards a geography of fitness: an ethnographic case study of the gym in British bodybuilding culture. Social Science \& Medicine, 60(4): 877-891.

[9] Biehler, D.D., Simon, L.G... (2010) The Great Indoors: research frontiers on indoor environments as active political-ecological spaces. Prog. Hum. Geogr. 35 (2), 172-192.

[10] Zenk, S. N., Schulz, A. J., Matthews, S. A., OdomsYoung, A., Wilbur, J. E., Wegrzyn, L., Gibbs, K., Braunschweig, C., Stokes, C. (2011) Activity space environment and dietary and physical activity behaviors: A pilot study. Health \& Place, 17, 11501161.

[11] Coen, Stephanie E, Davidson Joyce, Rosenberg. (2020)Towards a critical geography of physical activity: Emotions and the gendered boundarymaking of an everyday exercise environment. Transactions of the Institute of British Geographers, 45(2):313-330.

[12] Markula D P, Pringle R. (2007) Foucault, Sport and Exercise: Power, Knowledge and Transforming the Self. Taylor and Francis, Abingdon.

[13] Klein, J. (2001) Redefining the Body. PAJ: A Journal of Performance and Art, 23(1): 120-126.

[14] Berger J. (2009) Ways of seeing. Penguin Group, London.

[15] Waitt G. (2008) 'Killing waves': surfing, space and gender. Social \& Cultural Geography, 9(1):75-94. 